\title{
ASYMPTOTIC EQUIVALENCE OF SEQUENCES AND SUMMABILITY
}

\author{
JINLU LI \\ Department of Mathematics \\ Shawnee State University \\ Portsmouth, OH 45662
}

(Received August 14, 1995 and in revised form July 5, 1996)

ABSTRACT: For a sequence-to-sequence transformation $A$, let $R_{m} A x=\sum_{n \geq m}\left|(A x)_{n}\right|$ and $\mu_{m} A x=\sup _{n \geq m}\left|(A x)_{n}\right|$. The purpose of this paper is to study the relationship between the asymptotic equivalence of two sequences $\left(\lim _{n} x_{n} / y_{n}=1\right)$ and the variations of asymptotic equivalence based on the ratios $R_{m} A x / R_{m} A y$ and $\mu_{m} A x / \mu_{m} A y$.

KEY WORDS: Asymptotically regular, Asymptotic equivalence.

\section{AMS CLASSIFICATION CODES. PRIMARY $40 \mathrm{C05}$.}

\section{INTRODUCTION.}

Let $x=(x)_{n}$ and $y=(y)_{n}$ be infinite sequences, and let $A$ be a sequence-to-sequence transformation. We write $x \sim y$ if $\lim _{n} x_{n} / y_{n}=1$. In order to compare rates of convergence of sequences, in [2] Pobyvanets introduced the concept of asymptotically regular matrices, which preserve the asymptotic equivalence of two nonnegative sequences, that is $x \sim y$ implies $A x \sim A y$. Furthermore, in [1] Fridy introduced new ways to compare rates by using the ratios $R_{m} x / R_{m} y, \mu_{m} x / \mu_{m} y$ when they tend to zero. In [2] Marouf studied the relationship of these ratios when they have limit one. In the present study we investigate some further properties involved with the ratios such $\mu A x / \mu A y, R A x / R A y$ when they have limit one.

\section{NOTATIONS AND BASIC THEOREMS}

For a summability transformation $A$, we use $D_{A}$ to denote the domain of $A$ :

$$
D_{A}=\left\{x: \sum_{k=0}^{\infty} a_{n k} x_{k} \text { converges for such } n \geq 0\right\}
$$

and $C_{A}$ to denote the summability field:

$$
C_{A}=\left\{x: x \in D_{A}, \sum_{n=0}^{\infty} \sum_{k=0}^{\infty} a_{n_{k}} x_{k} \text { converges. }\right\}
$$

Also

$$
P_{\delta}=\left\{x: x_{n} \geq \delta>0 \text { for all } n\right\}
$$

and

$$
P=\left\{x: x_{n}>0 \text { for all } n .\right\}
$$

For a sequence $x$ in $\ell^{1}$ or $\ell^{\infty}$, we also define $R_{m} x=\sum_{n \geq m}\left|x_{n}\right|$ and $\mu_{m} x=\sup _{n \geq m}\left|x_{n}\right|$ for $m \geq 0$.

We list the following results without proof. 
THEOREM 1. (Pobyvanets [2]). A nonnegative matrix $A$ is asymptotically regular if and only if for each fixed interger $m, \lim _{n \rightarrow \infty} a_{n m} / \sum_{k=0}^{\infty} a_{n k} 0$.

THEOREM 2. A matrix $A$ is a $c_{0}-c_{0}$ matrix (i.e. A preserves zero limits) if and only if

(a) $\lim _{n \rightarrow \infty} a_{n k}=0$ for $k=0,1,2, \ldots$.

(b) There exists a number $M>0$ such that for each $n \sum_{k=0}^{\infty}\left|a_{n k}\right|<M$.

\section{ASYMPTOTIC EQUTVALENCE PROPERTIES.}

THEOREM 3. Let $A$ be a nonnegative matrix. Suppose $x \sim y$, and $x, y \in P_{\delta}$ for some $\delta>0$. Then $\mu A x \sim \mu A y$ if and only if for each $i=0,1,2, \ldots$

$$
\lim _{n \rightarrow \infty} a_{n i} / \sum_{i=0}^{\infty} a_{n j}=0
$$

PROOF. If $\lim _{n \rightarrow \infty} a_{n i} / \sum_{j=0}^{\infty} a_{n j}=0, i=0,1,2, \ldots$, we want to prove that $\mu A x \sim \mu A y$.

Since $x \sim y$, there exists a null sequence $\zeta$, such that

$$
x_{n}=y_{n}\left(1+\zeta_{n}\right) \quad n=0,1,2, \ldots
$$

then

$$
\begin{aligned}
\frac{(\mu A x)_{n}}{(\mu A y)_{n}} & =\frac{\sup _{k>n}(A x)_{k}}{\sup _{k \geq n}(A y)_{k}} \\
& =\frac{\sup _{k>n} \sum_{i=0}^{\infty} a_{k i} x_{i}}{\sup _{k \geq n} \sum_{i=0}^{\infty} a_{k i} y_{i}} \\
& =\frac{\sup _{k>n} \sum_{i=0}^{\infty} a_{k i}\left(y_{i}+y_{i} \zeta_{i}\right)}{\sup _{k \geq n} \sum_{i=0}^{\infty} a_{k i} y_{i}} \\
& \leq 1+\frac{\sup _{k \geq n} \sum_{i=0}^{\infty} a_{k i} y_{i}\left|\zeta_{i}\right|}{\sup _{k \geq n} \sum_{i=0}^{\infty} a_{k i} y_{i}} \\
& \leq 1+\frac{\sup _{k>n} \sum_{i=0}^{N} a_{k i} y_{i}\left|\zeta_{i}\right|}{\sup _{k \geq n} \sum_{i=0}^{\infty} a_{k i} y_{i}}+\frac{\sup _{k>n} \sum_{i=N+1}^{\infty} a_{k x} y_{i}\left|\zeta_{i}\right|}{\sup _{k \geq n} \sum_{i=0}^{\infty} a_{k i} y_{i}}
\end{aligned}
$$

where $N$ is a positive integer.

Since $\zeta$ is a null sequence, sup, $|\zeta|<,\infty$, and for any $\epsilon>0$ there is an $N \in N$, such that if $i \geq N$, then $\left|\zeta_{i}\right|<\epsilon$. Hence

$$
\begin{aligned}
\frac{(\mu A x)_{n}}{(\mu A y)_{n}} & \leq 1+\sup _{j}\left|\zeta_{j}\right| \sum_{i=0}^{N} \frac{\sup _{k>n} a_{k i} y_{i}}{\sup _{k \geq n} \sum_{i=0}^{\infty} a_{k i} y_{i}}+\frac{\epsilon \sup _{k \geq N} \sum_{i=N+1}^{\infty} a_{k i} y_{i}}{\sup _{k \geq n} \sum_{i=0}^{\infty} a_{k i} y_{i}} \\
& \leq 1+\sup _{j}\left|\zeta_{j}\right| \sum_{i=0}^{N} \frac{y_{i} \sup _{k>n} a_{k i}}{\delta \sup _{k \geq n} \sum_{i=0}^{\infty} a_{k i}}+\epsilon \\
& \leq 1+\sup _{j}\left|\zeta_{j}\right| \sup _{0 \leq j \leq N} y_{j} \sum_{i=0}^{N} \sup \frac{a_{k i}}{\sum_{i=0}^{\infty} a_{k i}}+\epsilon .
\end{aligned}
$$

According to the hypothesis, there exists $N_{1} \in \mathrm{N}$, such that if $k \geq N_{1}$, then $a_{k_{1}} / \sum_{i=0}^{\infty} a_{k_{t}}<$ $\epsilon / N \sup _{j} \zeta_{j} \sup _{0 \leq i \leq N} y_{i}$. So if $n \geq N$, we have 


$$
\frac{(\mu A x)_{n}}{(\mu A y)_{n}} \leq 1+\epsilon+\epsilon
$$

This implies that $\lim _{n \rightarrow \infty} \frac{(\mu A x)_{n}}{(\mu A y)_{n}} \leq 1$. Similarly, we may prove $\lim _{n \rightarrow \infty} \frac{\sup _{k \geq n} \sum_{i=0}^{\infty} a_{k i}}{\sup _{k \geq n} \sum_{i=0}^{\infty} a_{k 1}} \leq 1$ and the two inequalities yield $\lim _{n \rightarrow \infty} \frac{(\mu A x)_{n}}{(\mu A y)_{n}}=1$.

Next, suppose $\mu A x \sim \mu A y$ for any $x \sim y$ such that $x, y \in P_{\delta}$ for some $\delta>0$. We take $x=y=(1,1, \ldots)$. Then $\mu A x \sim \mu A y$, i.e.,

$$
\lim _{n \rightarrow \infty} \frac{\sup _{k>n} \sum_{i=0}^{\infty} a_{k i}}{\sup _{k \geq n} \sum_{i=0}^{\infty} a_{k i}}=1 .
$$

Hence, there exists $M>0$, such that $\left\{\sum_{i=0}^{\infty} a_{k i}\right\}_{k=0}^{\infty}$ is bounded by $M$.

If $\lim _{n \rightarrow \infty} a_{n i} / \sum_{j=0}^{\infty} a_{n}, \neq 0$ for some $i$. Then there exists $\lambda>0$ and $a$ sequence $n_{1}<n_{2}<\ldots$, such that $a_{u_{1}} / \sum_{j=0}^{\infty} a_{u}, \geq \lambda, u=1,2,3, \ldots$. Take $t>0$, and define $x$ and $y$ by

$$
y_{n}=1, n=0,1,2, \ldots
$$

and

$$
x_{n}= \begin{cases}1 & \text { if } n \neq \imath \\ 1+t & \text { if } n=i\end{cases}
$$

It is clear that $x \sim y$ and $x, y \in P_{1}$. Consider the following limit:

$$
\begin{aligned}
\lim _{u \rightarrow \infty} & \frac{\sup _{k>u} \sum_{i=0}^{\infty} a_{n_{k}} x}{\sup _{k \geq u} \sum_{i=0}^{\infty} a_{n_{k} j} y_{j}} \\
= & \lim _{u \rightarrow \infty} \frac{\sup _{k>u}\left(\sum_{i=0}^{\infty} a_{n_{k} j}+t a_{n_{k^{k}}}\right)}{\sup _{k \geq u} \sum_{i=0}^{\infty} a_{n_{k} j}} \\
\geq & \lim _{u \rightarrow \infty} \frac{\sup _{k>u}\left(\sum_{i=0}^{\infty} a_{n_{k} j}+t \lambda \sum_{j=0}^{\infty} a_{n_{k}}\right)}{\sup _{k \geq u} \sum_{i=0}^{\infty} a_{n_{k} j}} \\
= & 1+t \lambda .
\end{aligned}
$$

We can choose $t=1 / \lambda$, which gives

$$
\lim _{u \rightarrow \infty} \frac{(\mu A x)_{n_{u}}}{(\mu A y)_{n_{u}}} \geq 2
$$

This is a contradiction of $\mu A x \sim \mu A y$.

THEOREM 4. Suppose $A$ is a nonnegative matrix; then $\mu x \sim \mu y$ implies $\mu A x \sim \mu A y$ for any bounded sequences $x, y \in P_{\delta}$, for some $\delta>0$, if and only if $A$ satisfies the following three conditions:

(i) $\left(\sum_{j=0}^{\infty} a_{k j}\right)_{k=0}^{\infty}$ is a bounded sequence dominated by some $B$;

(ii) For any $j=0,1,2, \ldots$

$$
\lim _{n \rightarrow \infty} \frac{\sup _{k>n} a_{k j}}{\sup _{k \geq n} \sum_{i=0}^{\infty} a_{k i}}=0
$$

(iii) For any infinite sequence $j_{1}<j_{2}<j_{3} \ldots$ 


$$
\lim _{n \rightarrow \infty} \frac{\sup _{k>n} \sum_{i=1}^{\infty} a_{k j_{1}}}{\sup _{k \geq n} \sum_{i=0}^{\infty} a_{k j}}=1
$$

Before we prove this theorem, we shall give some examples of $A$ which satisfy the above conditions (i), (ii), and (iii).

Example 1. $A=I$.

Example 2.

$$
A=\left(\begin{array}{lllllll}
1 & & & & & \\
\frac{1}{2^{2}} & 1 & & & & \\
\frac{1}{3^{2}} & \frac{1}{3^{2}} & 1 & & 0 & \\
\frac{1}{4^{2}} & \frac{1}{4^{2}} & \frac{1}{4^{2}} & 1 & & \\
\cdots & & & \ddots & & \\
\frac{1}{(n+1)^{2}} & \frac{1}{(n+1)^{2}} & \cdots & \frac{1}{(n+1)^{2}} & 1 & \ddots
\end{array}\right)
$$

PROOF OF THEOREM 4. First, assume that for any bounded sequences $x, y \in P_{\delta}$, for some $\delta>0, \mu x \sim \mu y$ implies $\mu A x \sim \mu A y$; we wish to prove that $A$ satisfies the conditions (i), (ii) and (iii). Take $x=y=(1,1, \ldots)$; then $x, y$ are bounded, $x, y \in P_{1}$, and $\mu x \sim \mu y$; so $\mu A x \sim \mu A y$. But $(\mu A x)_{n}=\sup _{k \geq n} \sum_{j=0}^{\infty} a_{k j}$. Hence, $\left(\sum_{j=0}^{\infty} a_{k j}\right)_{k=0}^{\infty}$ should be bounded. This proves (i). To prove (ii) suppose there is a $j$ such that

$$
\varlimsup_{n \rightarrow \infty} \frac{\sup _{k>n} a_{k j}}{\sup _{k \geq n} \sum_{i=0}^{\infty} a_{k t}}=\lambda
$$

for some $\lambda>0$. As in the proof of Theorem 3, take $t>0$ and define $y=(1,1 \ldots)$ and

$$
x_{n}= \begin{cases}1 & \text { if } n \neq j \\ 1+t & \text { if } n=j\end{cases}
$$

Then $x, y \in P_{1}, x, y$ are bounded, and $\mu x \sim \mu y$; so we have $\mu A x \sim \mu A y$. But

$$
\begin{aligned}
& \varlimsup_{n \rightarrow \infty} \frac{\sup _{k>n} \sum_{i=0}^{\infty} a_{k} x_{i}}{\sup _{k \geq n} \sum_{i=0}^{\infty} a_{k i} y_{i}} \\
= & \varlimsup_{n \rightarrow \infty} \frac{\sup _{k>n}\left(t a_{k j}+\sum_{i=0}^{\infty} a_{k j}\right)}{\sup _{k \geq n} \sum_{i=0}^{\infty} a_{k i}} \\
\geq & \varlimsup_{n \rightarrow \infty} \frac{t \sup _{k>n} a_{k j}}{\sup _{k \geq n} \sum_{i=0}^{\infty} a_{k i}}-1 \\
= & t \lambda-1 .
\end{aligned}
$$

By choosing $t=\frac{3}{\lambda}$, we get

$$
\varlimsup_{n \rightarrow \infty} \frac{\sup _{k \geq n} \sum_{i=0}^{\infty} a_{k j} x_{i}}{\sup _{k \geq n} \sum_{i=0}^{\infty} a_{k i} x_{i}} \geq 3-1=2 .
$$

This is a contradiction $\mu A x \sim \mu A y$, so (ii) must hold.

Finally, we are going to prove (iii). For any given infinite sequence $j_{1}<j_{2}<\ldots$, we define $x$ and $y$ by

$$
y_{n}=2 \text { for every } n
$$

and

$$
x_{n}= \begin{cases}2, & \text { if } n=j_{u} \text { for } u=1,2, \ldots \\ 1, & \text { otherwise }\end{cases}
$$


It is easy to see that $x, y$ are bounded, $x, y \in P_{1}$ and $\mu x \sim \mu y$. This implies $\mu A x \sim \mu A y$. Hence we have

$$
\begin{aligned}
1 & =\lim _{n \rightarrow \infty} \frac{\sup _{k \geq n} \sum_{j=0}^{\infty} a_{k j} x_{j}}{\sup _{k \geq n} \sum_{j=0}^{\infty} a_{k \jmath} y_{\jmath}} \\
& =\lim _{n \rightarrow \infty} \frac{\left.\sup _{k>n} \sum_{, \in J} a_{k j} x,+\sum_{\jmath \in J} a_{k} x_{\jmath}\right)}{2 \sup _{k \geq n} \sum_{j=0}^{\infty} a_{k j}}
\end{aligned}
$$

where $J=\left\{j_{1}, j_{2}, j_{3}, \ldots\right\}$

$$
\begin{aligned}
& =\lim _{n \rightarrow \infty} \frac{\sup _{k>n}\left(2 \sum_{j \in J} a_{k J}+\sum_{j \in J} a_{k J}\right)}{2 \sup _{k \geq n} \sum_{j=0}^{\infty} a_{k j}} \\
& =\lim _{n \rightarrow \infty} \frac{\left(\sup _{k>n}\left(\sum_{j \in J} a_{k j}+\sum_{j=0}^{\infty} a_{k J}\right)\right.}{2 \sup _{k \geq n} \sum_{j=0}^{\infty} a_{k j}} \\
& \leq \lim _{n \rightarrow \infty} \frac{\sup _{k \geq n} \sum_{j \in J} a_{k j}+\sup _{k>n} \sum_{j=0}^{\infty} a_{k j}}{2 \sup _{k \geq n} \sum_{j=0}^{\infty} a_{k j}} \\
& =\lim _{n \rightarrow \infty} \frac{\sup _{k \geq n} \sum_{j \in J} a_{k J}}{2 \sup _{k \geq n} \sum_{j=0}^{\infty} a_{k j}}+\frac{1}{2} .
\end{aligned}
$$

Hence

This implies

$$
1 \leq \frac{1}{2} \lim _{n \rightarrow \infty} \frac{\sup _{k>n} \sum_{j \in J} a_{k j}}{\sup _{k \geq n} \sum_{j=0}^{\infty} a_{k}}+\frac{1}{2}
$$

$$
\lim _{n \rightarrow \infty} \frac{\sup _{k>n} \sum_{j \in J} a_{k j}}{\sup _{k \geq n} \sum_{j=0}^{\infty} a_{k j}} \geq 1
$$

On the other hand, it is clear that

$$
\lim _{n \rightarrow \infty} \frac{\sup _{k \geq n} \sum_{j \in J} a_{k J}}{\sup _{k \geq n} \sum_{j=0}^{\infty} a_{k j}} \leq 1
$$

Combining the last two inequalities together, we get

$$
\lim _{n \rightarrow \infty} \frac{\sup _{k>n} \sum_{\jmath \in J} a_{k j}}{\sup _{k \geq n} \sum_{\jmath=0}^{\infty} a_{k \jmath}}=1
$$

which proves (iii).

Conversely, assume $A$ satisfies the conditions (i), (ii) and (iii), and suppose $x, y$ are bounded by some $M>0, x, y \in P_{\delta}$ for some $\delta>0$, and $\mu x \sim \mu y$. For any $\epsilon>0$, since $x, y$ are bounded, there exists $N_{1} \in N$ such that if $j \geq N_{1}$, then

$$
y_{i} \leq \lim _{k \rightarrow \infty} \sup _{i \geq k} y_{i}+\epsilon
$$

and also there exists an infinite sequence $j_{1}<j_{2}<\ldots$, such that

$$
x_{j_{1}} \geq \lim _{k \rightarrow \infty} \sup _{j \geq k} x_{j}-\epsilon
$$

for $i=1,2,3, \ldots$. Therefore

$$
\lim _{n \rightarrow \infty} \frac{\sup _{k \geq n} \sum_{j=0}^{\infty} a_{k} x}{\sup _{k \geq n} \sum_{j=0}^{\infty} a_{k} y}
$$


$\geq \lim _{n \rightarrow \infty} \frac{\sup _{k>n} \sum_{i=0}^{\infty} a_{k j_{1}} x_{j_{1}}}{\sup _{k \geq n} \sum_{j=0}^{N_{1}} a_{k} y_{j}+\sum_{j=N_{1}+1}^{\infty} a_{k j} y_{j}}$

$\geq \lim _{n \rightarrow \infty} \frac{\sup _{k>n} \sum_{i=0}^{\infty} a_{k j_{1}}\left(\lim _{\ell \rightarrow \infty} \sup _{i \geq \ell} x_{i}-\epsilon\right)}{M \sup _{k \geq n} \sum_{\jmath=0}^{N_{1}} a_{k}+\sup _{k \geq n} \sum_{j=N_{1}+1}^{\infty} a_{k}\left(\lim _{\ell \rightarrow \infty} \sup _{\mathfrak{i} \geq \ell} y_{j}+\epsilon\right)}$

$\geq \lim _{n \rightarrow \infty} \frac{\left(\sup _{k>n} \sum_{i=0}^{\infty} a_{k j_{1}}\right) \lim _{\ell \rightarrow \infty} \sup _{i>\ell} x_{i}-\epsilon \sup _{k>n} \sum_{i=1}^{\infty} a_{k j_{1}}}{M \sup _{k \geq n} \sum_{j=0}^{N_{1}} a_{k j}+\epsilon \sup _{k \geq n} \sum_{j=0}^{\infty} a_{k j}+\sup _{k \geq n}\left(\sum_{j=N_{1}+1}^{\infty} a_{k j}\right) \lim _{\ell \rightarrow \infty} \sup _{\mathfrak{1}} \geq \ell}$

$\geq \lim _{n \rightarrow \infty} \frac{\left(\sup _{k \geq n} \sum_{i=0}^{\infty} a_{k j_{j}}\right) \lim _{\ell \rightarrow \infty} \sup _{i \ell \ell} x_{i}}{M \sup _{k \geq n} \sum_{\jmath=0}^{N_{1}} a_{k j}+\epsilon \sup _{k \geq n} \sum_{j=0}^{\infty} a_{k j}+\left(\sup _{k \geq n} \sum_{j=N_{1}+1}^{\infty} a_{k \jmath}\right) \lim _{\ell \rightarrow \infty} \sup _{i \geq \ell} y}$

$-\lim _{n \rightarrow \infty} \frac{\epsilon \sup _{k>n} \sum_{i=0}^{\infty} a_{k j_{1}}}{\left(\sup _{k \geq n} \sum_{N_{1}}^{\infty} a_{k j}\right) \lim _{\ell \rightarrow \infty} \sup _{i \geq \ell} y_{j}}$

$\geq \lim _{n \rightarrow \infty} \frac{\left(\sup _{k \geq n} \sum_{i=0}^{\infty} a_{k j_{1}}\right) \lim _{\ell \rightarrow \infty} \sup _{i>\ell} x_{i}}{M \sup _{k \geq n} \sum_{j=0}^{N_{1}} a_{k j}+\epsilon \sup _{k \geq n} \sum_{j=0}^{\infty} a_{k j}+\left(\sup _{k \geq n} \sum_{j=N_{1}+1}^{\infty} a_{k j}\right) \lim _{\ell \rightarrow \infty} \sup _{i \geq \ell} y_{j}}$

$-\frac{\epsilon}{\delta}$

(here, we used (iii) to deduce that

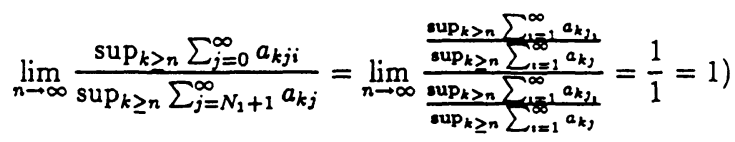

$$
\begin{aligned}
& \geq \lim _{n \rightarrow \infty} \frac{1}{B_{1}+B_{2}+B_{3}}-\frac{\varepsilon}{\delta}
\end{aligned}
$$

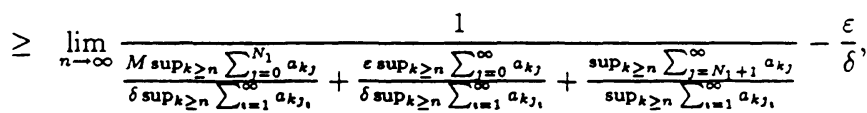

where

$$
\begin{aligned}
& B_{1}=\frac{M \sup _{k \geq n} \sum_{i=0}^{N_{1}} a_{k},}{\left(\sup _{k \geq n} \sum_{i=1}^{\infty} a_{k j_{1}}\right) \lim _{\ell \rightarrow \infty} \sup _{i \geq \ell} x_{i}}, B_{2}=\frac{\varepsilon \sup _{k \geq n} \sum_{j=0}^{\infty} a_{k j}}{\left(\sup _{k \geq n} \sum_{i=1}^{\infty} a_{k j_{i}}\right) \lim _{\ell \rightarrow \infty} \sup _{\imath \geq 1} x_{i}}, \\
& B_{3}=\frac{\left(\sup _{k \geq n} \sum_{j=N_{1}+1}^{\infty} a_{k j}\right) \lim _{\ell \rightarrow \infty} \sup _{i \geq \ell} y_{i}}{\left(\sup _{k \geq n} \sum_{i=1}^{\infty} a_{k j_{1}}\right) \lim _{\ell \rightarrow \infty} \sup _{i \geq \ell} x_{i}} .
\end{aligned}
$$

For the fixed $N_{1}$, combining conditions (ii) and (iii), we can easily prove

$$
\frac{\sup _{k>n} \sum_{j=0}^{N_{1}} a_{k j}}{\sup _{k \geq n} \sum_{i=1}^{\infty} a_{k j_{i}}} \rightarrow 0 \text { as } n \rightarrow \infty \text {. }
$$

Hence, for the given $\varepsilon>0$, there is $N_{2} \in \mathbf{N}$, such that if $n \geq N_{2}$, then

$$
\frac{\sup _{k \geq n} \sum_{j=0}^{N_{1}} a_{k j}}{\sup _{k \geq n} \sum_{i=1}^{\infty} a_{j_{1}}}<\varepsilon,
$$




$$
\frac{\sup _{k \geq n} \sum_{j=0}^{\infty} a_{k j}}{\sup _{k \geq n} \sum_{i=1}^{\infty} a_{k j}}<1+\varepsilon \quad(\text { by (iii)) }
$$

and

$$
\frac{\sup _{k \geq n} \sum_{j=0}^{N_{1}} a_{k j}}{\sup _{k \geq n} \sum_{i=1}^{\infty} a_{k j}}<1+\varepsilon \quad \text { (by (iii)) }
$$

These imply that if $n \geq N_{2}$

$$
\frac{1}{\frac{M \sup _{k \geq n} \sum_{j=0}^{N_{1}} a_{k}}{\delta \sup _{k \geq n} \sum_{i=1}^{\infty} a_{k j}}+\frac{\epsilon \sup _{k \geq n} \sum_{j=0}^{\infty} a_{k j}}{\delta \sup _{k \geq n} \sum_{i=1}^{\infty} a_{k} j_{1}}+\frac{\sup _{k \geq n} \sum_{j=N_{1}+1}^{\infty} a_{k j}}{\sup _{k \geq n} \sum_{i=1}^{\infty} a_{k_{j}}}} \geq \frac{1}{\frac{M \epsilon}{\delta}+\frac{e}{\delta}(1+\varepsilon)+1+\varepsilon} .
$$

Hence

$$
\lim _{n \rightarrow \infty} \frac{\sup _{k \geq n} \sum_{j=0}^{\infty} a_{k j} x_{j}}{\sup _{k \geq n} \sum_{j=0}^{\infty} a_{k j} y_{j}} \geq \frac{1}{\frac{M \epsilon}{\delta}+\frac{\varepsilon}{\delta}(1+\varepsilon)+1+\varepsilon}-\frac{\varepsilon}{\delta} .
$$

Since $\varepsilon$ is arbitrary, we have

$$
\lim _{n \rightarrow \infty} \frac{\sup _{k>n} \sum_{j=0}^{\infty} a_{k j} x_{j}}{\sup _{k \geq n} \sum_{j=0}^{\infty} a_{k j} y_{j}} \geq 1
$$

Similarly, we can prove

$$
\lim _{n \rightarrow \infty} \frac{\sup _{k \geq n} \sum_{j=0}^{\infty} a_{k j} x}{\sup _{k \geq n} \sum_{j=0}^{\infty} a_{k j} y} \leq 1 .
$$

Thus, we have finished the proof.

REMARK.

Let $A$ be a nonnegative matrix, $A=\left(a_{i}\right)$. If $A$ satisfies the following two conditions, then $A$ satisfies the conditions (i), (ii), (iii) of theorem 4:

a) There exists $\lambda>0$, such that

$$
\lim _{n \rightarrow \infty} a_{n n}=\lambda
$$

b) $\lim _{n \rightarrow \infty} \sum_{j \neq n} a_{n j}=0$

PROOF OF THE REMARK. If $A$ satisfies the above conditions a and $b$, it is easy to see that $A$ satisfies (i) in theorem 4. To prove (iii), let $j_{1}, j_{2}, \ldots$ be an infinity sequence: $j_{1}<j_{2}<\ldots$ Then

$$
\begin{aligned}
& \lim _{n \rightarrow \infty} \frac{\sup _{k>n} \sum_{i=1}^{\infty} a_{k j_{1}}}{\sup _{k \geq n} \sum_{j=0}^{\infty} a_{k j}} \geq \lim _{n \rightarrow \infty} \frac{\sup _{y_{1} \geq n} a_{j_{1} j_{1}}}{\sup _{k \geq n} \sum_{j=0}^{\infty} a_{k j}} \\
= & \frac{\lim _{n \rightarrow \infty} \sup _{j_{1} \geq n} a_{j_{1} j_{1}}}{\lim _{n \rightarrow \infty} \sup _{k \geq n} \sum_{j=0}^{\infty} a_{k j}}=\frac{\lambda}{\lambda+0}=1
\end{aligned}
$$

This inequality gives that

$$
\lim _{n \rightarrow \infty} \frac{\sup _{k>n} \sum_{i=1}^{\infty} a_{k j_{1}}}{\sup _{k \geq n} \sum_{j=0}^{\infty} a_{k j}}=1
$$

Next, let's prove (ii) of theorem 4 . In fact, for any fixed $j=0,1,2, \ldots$

$$
\begin{aligned}
& \lim _{n \rightarrow \infty} \frac{\sup _{k>n} a_{k j}}{\sup _{k \geq n} \sum_{i=0}^{\infty} a_{k j}} \\
\leq & \lim _{n \rightarrow \infty} \frac{\sup _{k>n} \sum_{j<k} a_{k j}}{a_{n n}} \\
= & \frac{\lim _{n \rightarrow \infty} \sup _{k>n} \sum_{j<k} a_{k j}}{\lim _{n \rightarrow \infty} a_{n n}}
\end{aligned}
$$




$$
\begin{aligned}
& \leq \frac{\lim _{n \rightarrow \infty} \sup _{k>n} \sum_{\jmath \neq k} a_{k j}}{\lambda} \\
& =\frac{\lim _{n \rightarrow \infty} \sum_{j \neq n} a_{n \jmath}}{\lambda} \\
& =0
\end{aligned}
$$

Next, we give some examples to show that the conditions of theorem 4 are necessary. Example 3. Let $A$ be defined as follows:

$$
A=\left(\begin{array}{llllllllll}
2 & 0 & 2 & 0 & 0 & 0 & 0 & 0 & 0 & \\
& 1 & 2 & 0 & 0 & 0 & 0 & 0 & 0 & \\
& & 2 & 0 & 2 & 0 & 0 & 0 & 0 & \\
& & & 1 & 2 & 0 & 0 & 0 & 0 & \\
& & & & 2 & 0 & 2 & 0 & 0 & \\
& & & & & & 1 & 2 & 0 & 0 \\
& & & & & & & \\
& & & & & & 2 & 0 & 2 & \\
& 0 & & & & & & 1 & 2 & \\
& & & & & & & & 2 & \\
& & & & & & & & & \\
& & & & & & & & & \ddots
\end{array}\right)
$$

It is easy to see that $A$ satisfies the conditions (i) and (ii), not (iii).

Take

$$
\begin{aligned}
& x=(2,2,2,2, \ldots) \\
& y=(2,1,1,1,2,1,1,1,1,2,1,1,1,1,2, \ldots)
\end{aligned}
$$

$x, y$ are bounded sequences and $x, y \in P_{1}$. For $m=1,2,3, \ldots$ we have $\mu_{m}(x)=\mu_{m}(y)=2$. Hence $\frac{\mu_{m}(x)}{\mu_{m}(y)}=1$. But

$$
\begin{aligned}
& A x=(8,8,8, \ldots) \\
& A y=(6,3, \ldots) \quad y=\left(y_{\mathrm{\imath}}\right) \quad y_{\mathrm{t}} \leq 6 \quad i=1,2, \ldots
\end{aligned}
$$

This implies

$$
\frac{\mu_{m} A x}{\mu_{m} A y} \rightarrow \frac{8}{6}=\frac{4}{3} \neq 1 \text {, as } n \rightarrow \infty \text {. }
$$

Example 4.

Let

$$
A=\left(\begin{array}{cccccc}
1 & & & & \\
& \frac{1}{2} & & & 0 & \\
& & \frac{1}{4} & & & \\
& & & \frac{1}{8} & & \\
& & & & \ddots \\
& & 0 & & &
\end{array}\right) .
$$

$A$ satisfies (i) and (ii) not (iii).

Take

$$
\begin{aligned}
& x=(2,2,2, \ldots) \\
& y=(2,1,2,1, \ldots) .
\end{aligned}
$$


$x$ and $y$ are bounded and $x, y \in P_{1}$. We also have

$$
\begin{gathered}
\frac{\mu_{m} x}{\mu_{m} y}=1, m=1,2, \ldots \\
A x=\left(2,1, \frac{1}{2}, \frac{1}{4}, \frac{1}{8}, \ldots\right) \\
A y=\left(2, \frac{1}{2}, \frac{1}{2}, \frac{1}{4}, \frac{1}{4}, \frac{1}{8}, \frac{1}{8}, \ldots\right) .
\end{gathered}
$$

Then, if $m$ is odd,

$$
\frac{(\mu A x)_{m}}{(\mu A y)_{m}}=2
$$

if $m$ is even

$$
\frac{(\mu A x)_{m}}{(\mu A y)_{m}}=1
$$

$\Rightarrow \frac{(\mu A x)_{m}}{(\mu A y)_{m}}$ has no limit as $m \rightarrow \infty$.

ACKNOWLEDGEMENT The author appreciates Professor J.A. Fridy for his communication and many useful suggestions.

\section{REFERENCES}

[1] Fridy, J.A. Minimal Rates of Summability. Can. J. Math., Vol. XXX, 4 (1978), 808-816.

[2] Marouf, M. S. Asymptotic Equivalence And Summability. Internat. J. Math \& Math. Sci. Vol. 16 No. 4 (1993) 755-762.

[3] Pobyvanets, I.P. Asymptotic Equivalence of Some Linear Transformation Defined by a Nonnegative Matrix and Reduced to Generalized Equivalences in the Sense of Cesáro and Abel, Mat. Fig. 28 (1980), 83-87, 123.

[4] Powel, R.E. and Shah, S.E. Summability Theory and its Applications, Van Nostrand Rienhold London, 1972. 


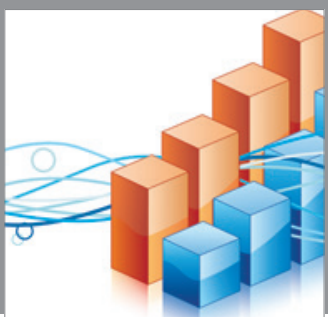

Advances in

Operations Research

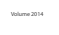

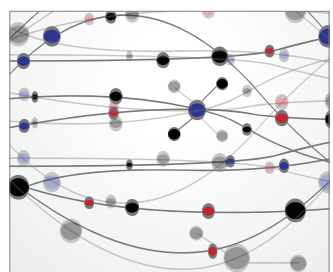

\section{The Scientific} World Journal
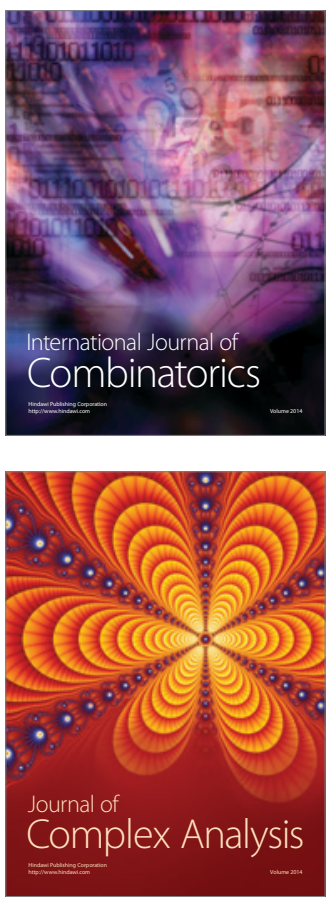

International Journal of

Mathematics and

Mathematical

Sciences
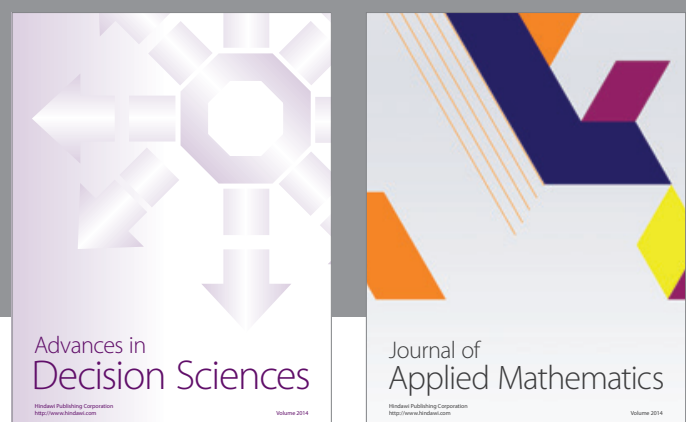

Journal of

Applied Mathematics
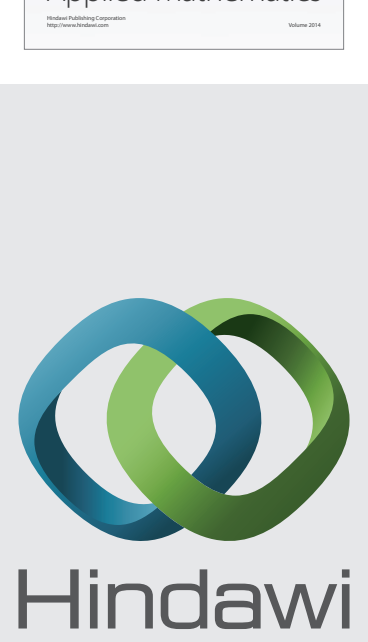

Submit your manuscripts at http://www.hindawi.com
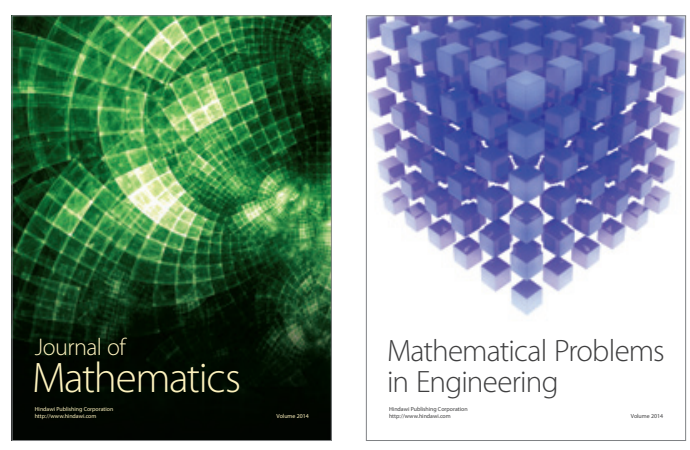

Mathematical Problems in Engineering
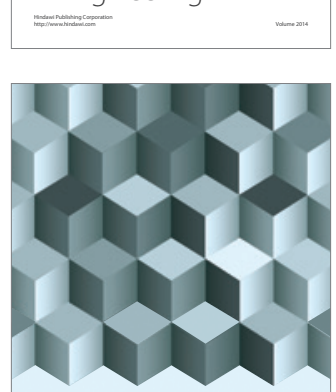

Journal of

Function Spaces
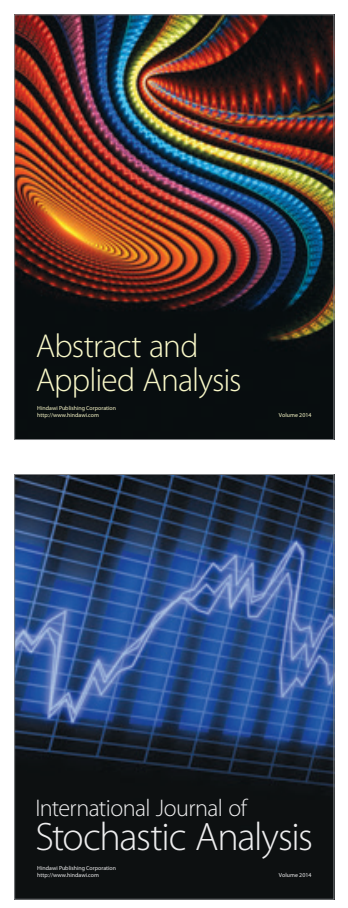

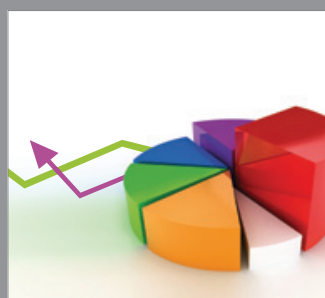

ournal of

Probability and Statistics

Promensencen
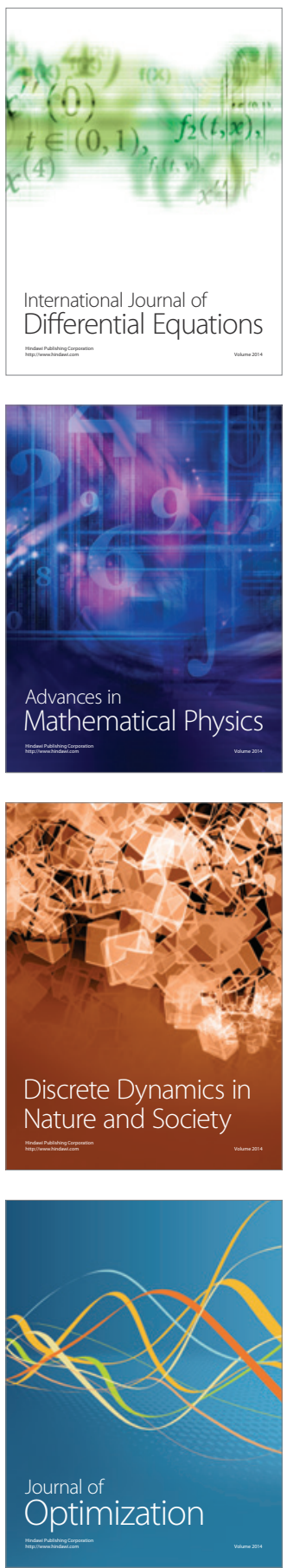